\title{
Spontaneous Transomental Hernia
}

\author{
Seung Hun Lee, Seung Hyun Lee \\ Department of Surgery, Kosin University Gospel Hospital, Kosin University College of Medicine, Busan, Korea
}

A transomental hernia through the greater or lesser omentum is rare, accounting for approximately $4 \%$ of internal hernias. Transomental hernias are generally reported in patients aged over fifty. In such instances, acquired transomental hernias are usual, are commonly iatrogenic, and result from surgical interventions or from trauma or peritoneal inflammation. In rare cases, such as the one described in this study, internal hernias through the greater or lesser omentum occur spontaneously as the result of senile atrophy without history of surgery, trauma, or inflammation. A transomental hernia has a high postoperative mortality rate of 30\%, and emergency diagnosis and treatment are critical. We report a case of a spontaneous transomental hernia of the small intestine causing intestinal obstruction. An internal hernia with strangulation of the small bowel in the lesser sac was suspected from the image study. After an emergency laparotomy, a transomental hernia was diagnosed.

Keywords: Omentum; Intestinal obstruction; Hernia

\section{INTRODUCTION}

An internal hernia occurs when viscera protrude through a congenital or acquired peritoneal or mesenteric aperture in the peritoneal cavity. Hernias account for $5.8 \%$ of intestinal obstruction cases [1-3]. Aperture types are paraduodenal (53\%), pericecal (13\%), foramen of Winslow (8\%), transmesenteric and transmesocolic (8\%), intersigmoid (6\%), retroanastomotic (5\%), and transomental (1\%-4\%) [4]. Transomental hernias are very rare and usually congenital; they are rarely traumatic or iatrogenic [5-7]. We report a case of a spontaneous transomental hernia of the small intestine causing intestinal obstruction.

\section{CASE REPORT}

A 57-year-old man was admitted to the Emergency Department with an acute abdomen. He reported abrupt onset of epigastric

Received: June 21,2015 • Accepted: January 27, 2016

Correspondence to: Seung Hyun Lee, M.D.

Department of Surgery, Kosin University Gospel Hospital, Kosin University

College of Medicine, 262 Gamcheon-ro, Seo-gu, Busan 49267, Korea

Tel: +82-51-990-6231, Fax: +82-51-246-6093

E-mail:gscrslsh@hanmail.net

(C) 2016 The Korean Society of Coloproctology

This is an open-access article distributed under the terms of the Creative Commons Attribution NonCommercial License (http://creativecommons.org/licenses/by-nc/4.0) which permits unrestricted noncommercial use, distribution, and reproduction in any medium, provided the original work is properly cited. pain and bilious vomiting with abdominal distension for at least the preceding 24 hours. The patient could not recall previous, similar pain attacks. He had no prior surgical procedures or history of abdominal trauma.

Vital signs were all within normal limits. On physical examination, the abdomen was mildly distended and tympanic on percussion. The abdomen showed epigastric tenderness, but not rebound tenderness, and had audible hyperactive bowel sounds. The patient had no visible abdominal surgical scars. Laboratory tests were unremarkable except for leukocytosis $\left(14.09 \times 10^{3} / \mathrm{mL}\right.$ with $88.8 \%$ neutrophils). A plain film of the abdomen demonstrated dilated small bowel loops with an air-fluid level in the epigastrium and on the right side of the abdomen, suggesting intestinal obstruction (Fig. 1). A computed tomography (CT) scan of the abdomen revealed dilated, hypo-enhancing small bowel loops with a swirling pattern in the mesenteric vessels surrounded to the right by the gallbladder and liver, to the left by the stomach, and posteriorly by the pancreas (Figs. 2, 3). An internal hernia with strangulation of the small bowel in the lesser sac was suspected.

An emergency laparotomy was performed. An approximately $50-\mathrm{cm}$ segment of the jejunum and the proximal ileum in the lesser sac was herniated through a $2.0-\mathrm{cm}$ slit-like defect in the distal part of the gastrocolic ligament (Fig. 4A, B). The incarcerated small bowel loops appeared congested, but did not strangulate. After reduction of small bowel loops, the ischemic signs of the herniated loops recovered. The omentum was divided. The postoperative course was uneventful. The patient was discharged 


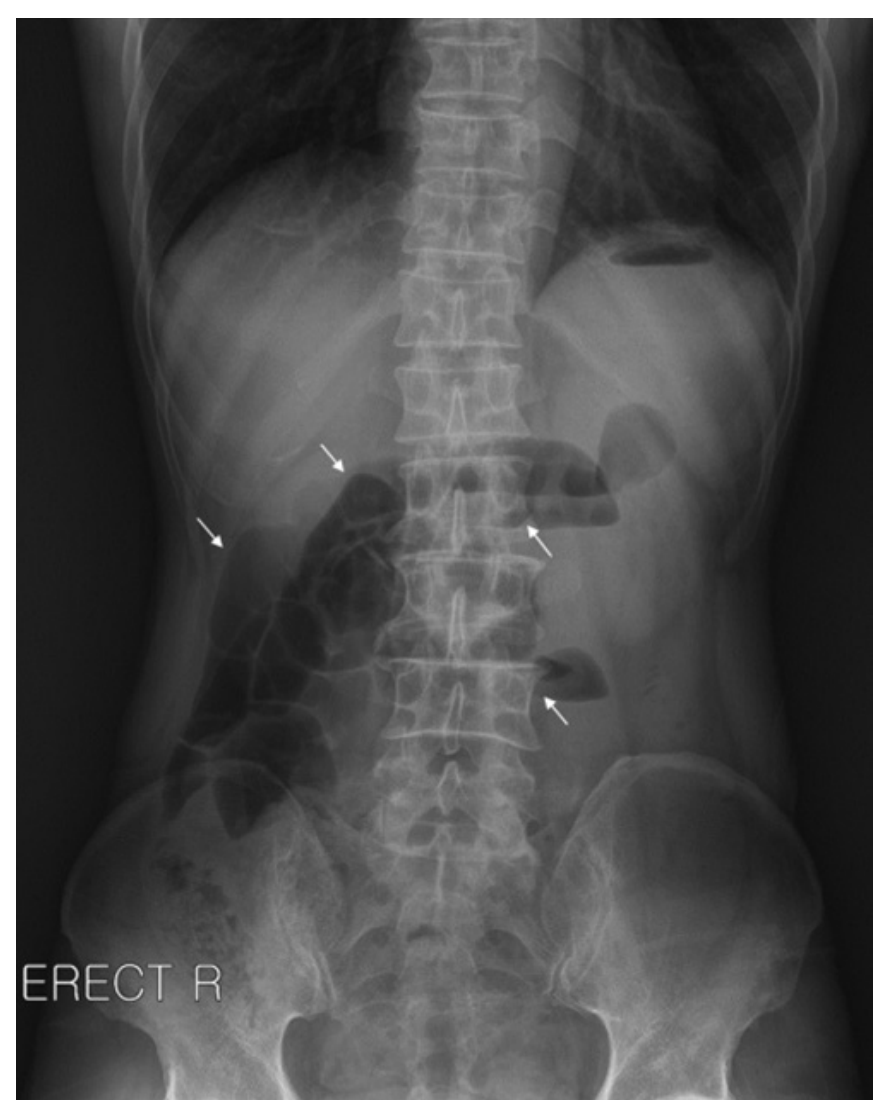

Fig. 1. Abdominal plain film shows dilated small bowel loops with an air-fluid level in the epigastrium and on the right side of the abdomen (arrows).

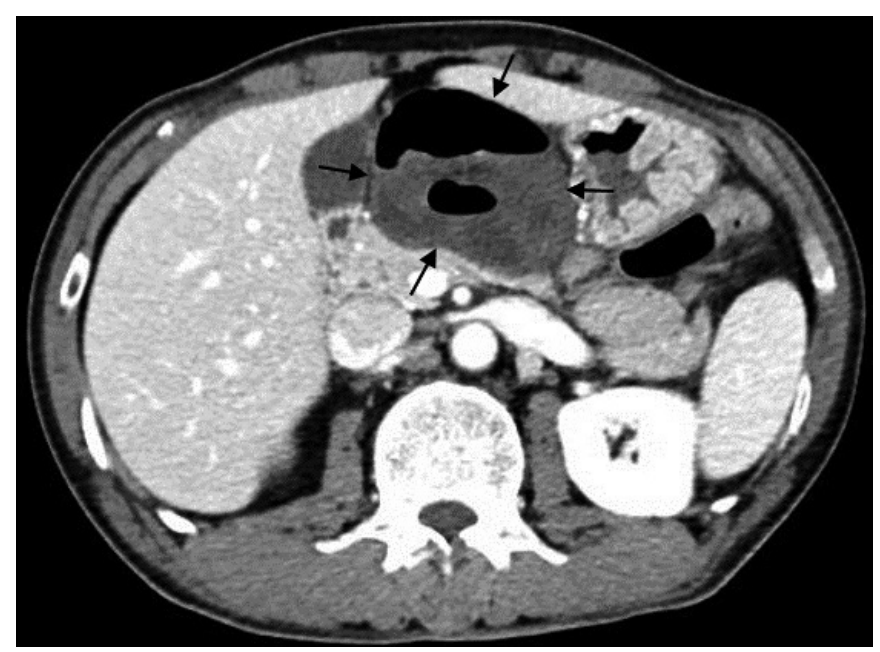

Fig. 2. Computed tomography scan (axial image at the level of the pancreas) shows a cluster of distended and hypo-enhancing small bowel loops surrounded by the gallbladder and liver to the right, by the stomach to the left, and by the pancreas posteriorly (arrows). An internal hernia with strangulation of the small bowel localized in the lesser sac is seen.

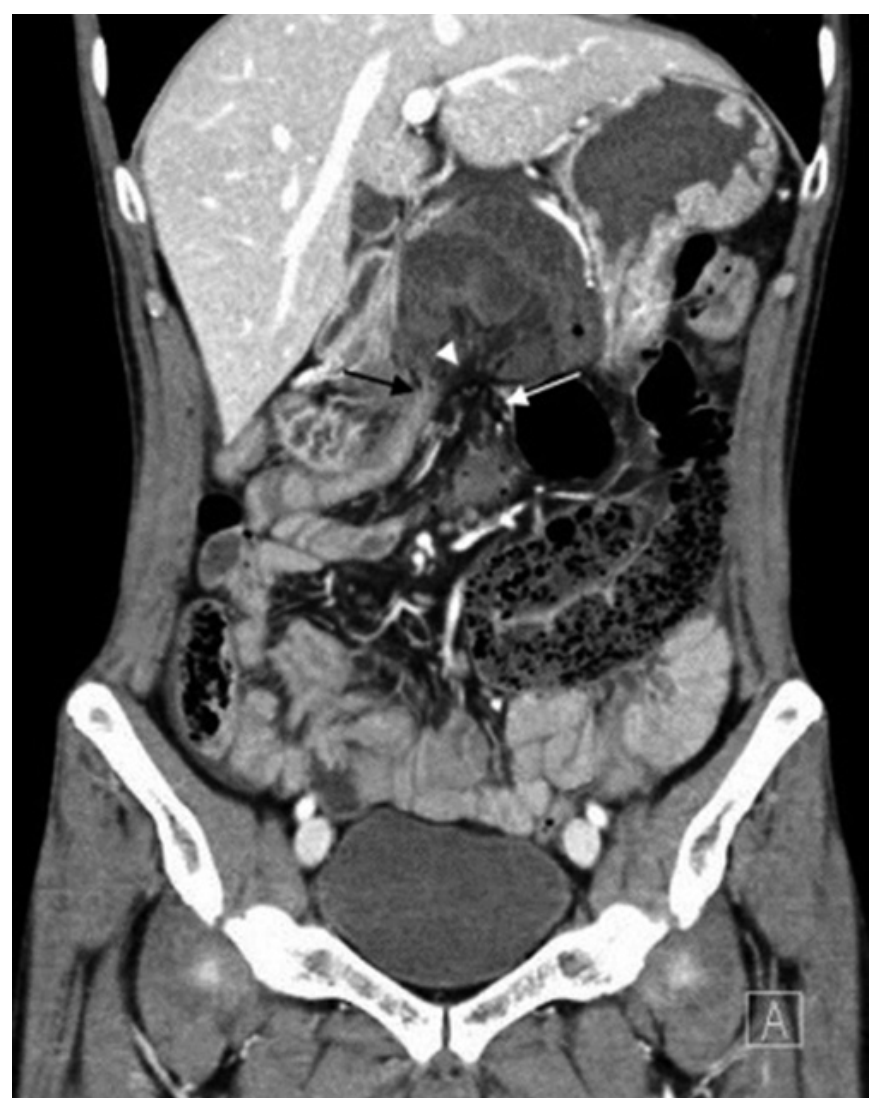

Fig. 3. Computed tomography scan (coronal image) shows a closely apposed afferent loop (white arrow) and efferent loop (black arrow) of the small bowel with a swirling pattern in the mesenteric vessels being appreciated along with the hernia orifice (arrowhead).

on the ninth day after entering the hospital.

\section{DISCUSSION}

Internal hernias are protrusions of the viscera through a peritoneal or mesenteric opening, with the herniated viscera remaining within the abdominal cavity. Openings have normal (foramen of Winslow), paranormal (paraduodenal, ileocecal, supravesical fossa), and abnormal (transomental defect) anatomies $[1,8]$. Based on autopsy studies, the overall incidence of internal hernias is $0.2 \%-0.9 \%$ [1]. Internal hernias constitute up to $0.6 \%-5.8 \%$ of all intestinal obstructions $[2,9]$. Hernia mortality may exceed $45 \%[2,9]$.

A transomental hernia through the greater or lesser omentum is rare, accounting for approximately $1 \%-4 \%$ of internal hernias [1]. Transomental hernias are generally reported in patients aged over fifty [10]. In such instances, acquired transomental hernias are usual, are commonly iatrogenic, and result from surgical interventions (Roux-end-Y gastric bypass, liver transplantation, and small bowel or colon resection) or from trauma or peritoneal in- 

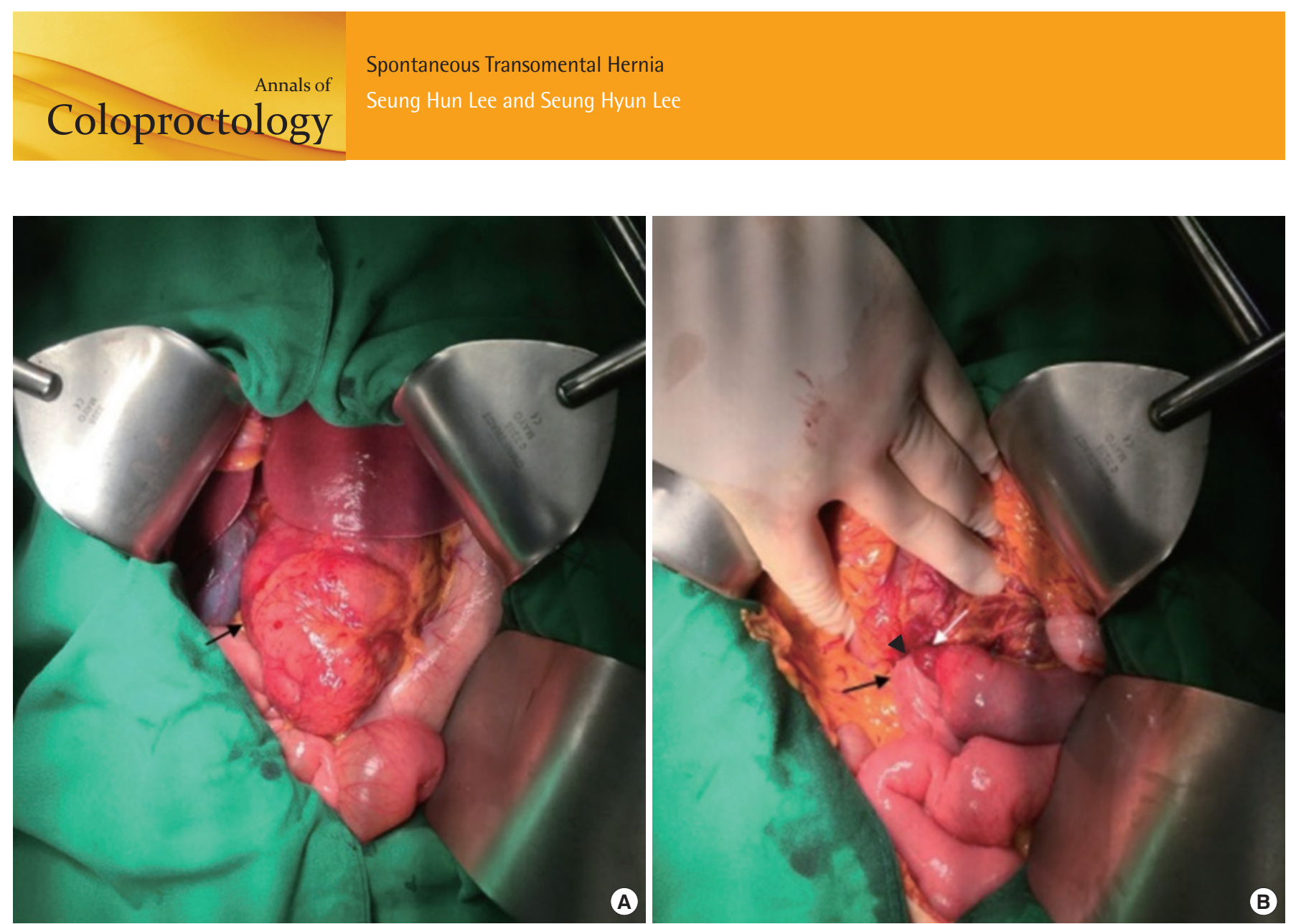

Fig. 4. Findings of an exploratory laparotomy: (A) a segment of the jejunum and the proximal ileum was located in the lesser sac (arrow) and (B) small bowel loops were herniated through a $2.0-\mathrm{cm}$ slit-like defect in the distal part of gastrocolic ligament (arrowhead). As shown on computed tomography, the afferent loop (white arrow) and the efferent loop (black arrow) of the small bowel were identified.

flammation [5-7]. In children, congenital transomental hernias are common and occur in males versus females at a ratio of about 2 to 1 [6]. In children, disease severity depends on the orifice size and the length of the herniated small bowel loops [11]. In rare cases, such as the one described in this study, internal hernias through the greater or lesser omentum occur spontaneously as the result of senile atrophy without history of surgery, trauma, or inflammation [10]. Yamaguchi [12] classified transomental hernias as type A (peritoneal cavity $\rightarrow$ greater omentum $\rightarrow$ peritoneal cavity), B (peritoneal cavity $\rightarrow$ omental bursa $\rightarrow$ peritoneal cavity), or C (peritoneal cavity $\rightarrow$ omental bursa). Type A is slightly more common than type $\mathrm{C}$, and type $\mathrm{A}$ is predominant in older patients [12]. Our case was type C.

Transomental hernias are difficult to diagnosis. Clinical manifestations are not specific and are similar to acute obstructive syndrome including nausea, vomiting, abdominal pain, distended abdomen, and constipation. Compared with other types of internal hernias, patients with transomental hernias present more frequently with strangulation of the small bowel. In most cases, a gangrenous bowel is present at the exploratory laparotomy [9]. For this reason, transomental hernias have a high postoperative mortality rate of $30 \%$, so emergency diagnosis and treatment are critical $[6,9]$.
Abdominal CT scans are helpful for diagnosis. Abdominal CT scans may reveal dilated small bowel loops with a "beak sign," which is a triangular configuration of the transition zone between the proximal dilated bowels and the herniated bowel segments or between dilated, herniated bowel segments and distal, collapsed bowel segments [7]. CT might also reveal a swirling pattern of the mesenteric vessels, engorged mesenteric vessels with a large impact on the surrounding organs and bowel wall thickening [13]. In addition, a transomental hernia should be suspected if the dilated small bowel loops are localized in the lesser sac (i.e., surrounded to the right by the gallbladder and liver, to the left by the stomach, and posteriorly by the pancreas) and the neck of the hernia orifice is seen as mesenteric vessels passing through the omental defect, with abdominal CT scans showing no evidence of loops passing through the foramen of Winslow between the inferior vena cava and the portal triad [14]. However, in most cases, a definitive diagnosis is established intraoperatively [14].

Surgical treatment is the reduction of the herniated intestinal segments. If necrosis, perforation, or irreversible ischemia of the herniated viscera is present, bowel resection is necessary. In sequence, the defect of omentum must be repaired to prevent subsequent herniation. Even without risk factors of previous abdominal surgery, trauma or peritoneal inflammation, the possibility of 
small bowel obstruction secondary to the internal hernia should be considered. Surgical treatment based on high clinical suspicion can reduce the risk of complications and postoperative mortality in patients with a transomental hernia.

\section{CONFLICT OF INTEREST}

No potential conflict of interest relevant to this article was reported.

\section{REFERENCES}

1. Ghahremani GG. Internal abdominal hernias. Surg Clin North Am 1984;64:393-406.

2. Ghahremani GG. Abdominal and pelvic hernias. In: Gore RM, Levine MS, editors. Textbook of gastrointestinal radiology. 2nd ed. Philadelphia (PA): Saunders; 2000. p. 1993-2009.

3. Martin LC, Merkle EM, Thompson WM. Review of internal hernias: radiographic and clinical findings. AJR Am J Roentgenol 2006; 186:703-17.

4. Meyers MA. Dynamic radiology of the abdomen: normal and pathologic anatomy. 4th ed. New York (NY): Springer-Verlag; 1994.

5. Blachar A, Federle MP. Internal hernia: an increasingly common cause of small bowel obstruction. Semin Ultrasound CT MR 2002;
23:174-83.

6. Hull JD 3rd. Transomental hernia. Am Surg 1976;42:278-84.

7. Delabrousse E, Couvreur M, Saguet O, Heyd B, Brunelle S, Kastler B. Strangulated transomental hernia: CT findings. Abdom Imaging 2001;26:86-8.

8. Stewart JO. Lesser sac hernia. Brit J Surg 1962;50:321-6.

9. Newsom BD, Kukora JS. Congenital and acquired internal hernias: unusual causes of small bowel obstruction. Am J Surg 1986; 152:279-85.

10. Yang DH, Chang WC, Kuo WH, Hsu WH, Teng CY, Fan YG. Spontaneous internal herniation through the greater omentum. Abdom Imaging 2009;34:731-3.

11. Chou CK, Mak CW, Wu RH, Chang JM. Combined transmesocolic-transomental internal hernia. AJR Am J Roentgenol 2005; 184:1532-4.

12. Yamaguchi T. A case of incarceration of sigmoid colon into hiatus of greater omentum. Rinsho Geka 1978;33:1041-5.

13. Camera L, De Gennaro A, Longobardi M, Masone S, Calabrese E, Del Vecchio W, et al. A spontaneous strangulated transomental hernia: prospective and retrospective multi-detector computed tomography findings. World J Radiol 2014;6:26-30.

14. Guinier D, Tissot O. Strangulated lesser sac hernia. J Visc Surg 2012;149:e221-2. 